\title{
Intususcepción ileotransversa por lipoma intraluminal
}

\author{
lleotransverse intussusception due to intraluminal lipoma
}

José Julián Valencia-Correa', Juan Esteban Botero-Velasquez², Santiago Salazar-Ochoa ${ }^{3 凶}$, Alejandro Montoya-Arboleda ${ }^{3}$, Laura Isabel Jaramillo-Duque ${ }^{4}$ CvLAC

Fecha correspondencia:

Recibido: enero 19 de 2019.

Revisado: junio 10 de 2019.

Aceptado: julio 3 de 2019.

Forma de citar:

Valencia-Correa JJ, Botero-

Velasquez JE, Salazar-Ochoa S,

Montoya-Arboleda A, Jaramillo-

Duque LI. Intususcepción

ileotransversa por lipoma

intraluminal. Rev CES Med.

2019;33(3): 224-230.

\section{Open access}

(C) Derecho de autor

Licencia creative commons

Ética de publicaciones

Revisión por pares

Gestión por Open Journal System

DOl: http://dx.doi.org/10.21615/

cesmedicina.33.3.8

ISSN 0120-8705

e-ISSN 2215-9177

Sobre los autores:

1. Radiólogo Clínica CES

y docente de Radiología

Universidad CES.

Comparte

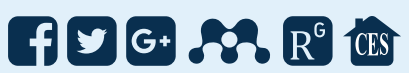

\section{Resumen}

La intususcepción intestinal consiste en la introducción o invaginación de un segmento del intestino dentro de la luz del segmento adyacente. Una parte del intestino se pliega como un telescopio y penetra dentro de otra. La mayoría de los casos se producen durante los primeros cinco años de vida y la mitad de estos durante el primer año. En los niños mayores y en los adultos frecuentemente es el resultado de lesiones tumorales intraluminales como lipomas, carcinoma, pólipos fibroides inflamatorios o linfoma maligno. Se presenta el caso clínico de una paciente de 45 años con cuadro de dolor abdominal originado por intususcepción ileotransversa causada por un lipoma intraluminal, el cual fue resuelto exitosamente mediante cirugía.

Palabras claves: Intususcepción; Lipoma; Íleon; Colon transverso; Colectomía.

\section{Abstract}

Intussusception involves the introduction or invagination of a segment of the intestine within the lumen of the adjacent segment. A part of the intestine folds like a telescope and penetrates into another. Most cases occur during the first five years of life and half of these during the first year. In older children and adults are the result of intraluminal tumor lesions such as lipomas, carcinoma, inflammatory fibroid polyps or malignant lymphoma. We present the clinical case of a 45-year-old patient with abdominal pain caused by ileotransverse intussusception caused by an intraluminal lipoma, which was successfully resolved by surgery.

Keywords: Intussusception; Lipoma; Ileum; Colon, transverse; Colectomy.

\section{Introducción}

La intususcepción se define como la introducción o invaginación de un segmento proximal del intestino dentro del segmento distal inmediato. Una vez adentro, el segmento invaginado es propulsado por la peristalsis y tracciona el mesenterio a lo largo de la invaginación, avanzando tanto como la contra tracción mesentérica se lo permite (1).

En niños menores de dos años la intususcepción es la causa más común de obstrucción intestinal. En ellos, la causa idiopática es usual y generalmente no existe defecto anatómico subyacente; otras causas asociadas a este cuadro son las infecciones virales y las vacunas (para rotavirus parti- 
2. Cirujano general Clínica CES y docente de Cirugía Universidad CES.

3. Medico general.

4. Residente de Radiología Universidad CES.
En adultos, hasta un $90 \%$ de las intususcepciones son causadas por lesiones intraluminales las cuales sirven como punto inicial de tracción; aunque también puede darse por otras causas como idiopática, en postoperatorios de cirugías bariátricas, por apendicitis aguda y menos comúnmente por divertículos de Meckel invertidos. cularmente), quizás debido a la hiperplasia reactiva de las placas de Peyer y de otros tejidos linfoides asociados a la mucosa intestinal que pueden actuar como punta de lanza para la intususcepción (2).

En niños mayores y adultos es rara la intususcepción y representa menos del $5 \%$ de los casos; además, en este grupo poblacional la intususcepción es la causa de obstrucción intestinal solo en el 1-5 \% de los casos. En adultos, hasta un $90 \%$ de las intususcepciones son causadas por lesiones intraluminales las cuales sirven como punto inicial de tracción; aunque también puede darse por otras causas como idiopática, en postoperatorios de cirugías bariátricas, por apendicitis aguda y menos comúnmente por divertículos de Meckel invertidos (cuadro 1) (3-5).

Cuadro 1. Causas de intususcepción en adultos

\begin{tabular}{ll}
\hline \multicolumn{1}{c}{ Benignas } & \multicolumn{1}{c}{ Malignas } \\
\hline Lipomas & \\
Adenoma & \\
Enfermedad de Crohn & Adenocarcinoma \\
Endometriosis & Linfoma \\
Tumores del estroma & Sarcoma \\
gastrointestinal (GIST) & Tumor carcinoide \\
Hamartoma & Tumores del estroma \\
Hemangioma & gastrointestinal malignos \\
Diverticulo de Meckel & (GIST) \\
Polipo de Peutz-Jegher & Tumor neuroendocrino \\
Nuerofibroma & Leiomiosarcoma \\
Polipo inflamatorio & Carcinoma metastásico \\
Neurofibroma & (melanoma el mas común) \\
Tuberculosis & \\
Enfermedad celiaca & \\
\hline
\end{tabular}

Aunque es una entidad relativamente rara en la población adulta, constituye una entidad quirúrgica importante, con presentación clínica generalmente inespecífica, lo que lleva a que con frecuencia se realicen diagnósticos tardíos o intraoperatorios (b).

Se presenta el caso clínico de una paciente con una intususcepción ileotransversa. Además, se hace una revisión de la literatura actual disponible sobre la fisiopatología, presentación clínica, diagnóstico y manejo.

\section{Caso clínico}

Una mujer de 45 años, con antecedentes de hipotiroidismo tratado con levotiroxina, fumadora de 20 paquetes/año y una cesárea como antecedente quirúrgico, consultó por un cuadro de ocho días evolución consistente en dolor abdominal tipo cólico, de localización inicial en abdomen superior, que posteriormente se irradió al resto de este, refiriendo que en el último día el dolor aumentó en intensidad y frecuencia 
Una tomografía abdominal simple en decúbito prono evidenció intususcepción ileocecal con extensión hacia el colon transverso y lesión sólida que sugería lipoma intestinal en la cabeza de la intususcepción; sin signos de obstrucción intestinal. siendo calificado como 10/10 en la escala visual analógica del dolor. Refirió también náuseas, sin emesis. Reportaba cambios en el color de la orina (colúrica) y negaba deposiciones diarreicas u otros síntomas relevantes.

En la evaluación inicial presentaba signos vitales dentro de límites normales. Manifestaba dolor durante la palpación del hipocondrio derecho, aunque sin signos de irritación peritoneal. Por lo anterior se sospechó como principal causa del dolor una posible enfermedad hepatobiliar y se solicitaron pruebas de función hepática, bilirrubinas y análisis urinario.

Los resultados no evidenciaron alteraciones significativas mostrando únicamente una leve leucocitosis sin neutrofilia, por lo que se revaluó a la paciente y se evidenció aumento del dolor a la palpación en hemiabdomen inferior.

Se sospechó entonces que el dolor estuviera causado por una urolitiasis, por lo que se solicitó tomografía abdominal simple con protocolo en decúbito prono, en la que se evidenció imagen de intususcepción ileocecal con extensión hacia el colon transverso y lesión sólida con densidad grasa que sugería lipoma intestinal en la cabeza de la intususcepción; no evidenciándose signos de obstrucción intestinal (figura 1).

Se solicitó entonces valoración por cirugía general quienes explican a la paciente el procedimiento a realizar y, previo consentimiento informado sobre beneficios, riesgos y complicaciones proceden a realizar la cirugía con reducción laparoscópica de la intususcepción más hemicolectomía derecha abierta y anastomosis ileotransversa (figura 2). La paciente evolucionó adecuadamente en el posoperatorio y fue dada de alta. Posteriormente, se realiza seguimiento ambulatorio sin encontrar complicaciones asociadas y con resultado de biopsia que mostró lipoma submucoso ileal negativo para malignidad.

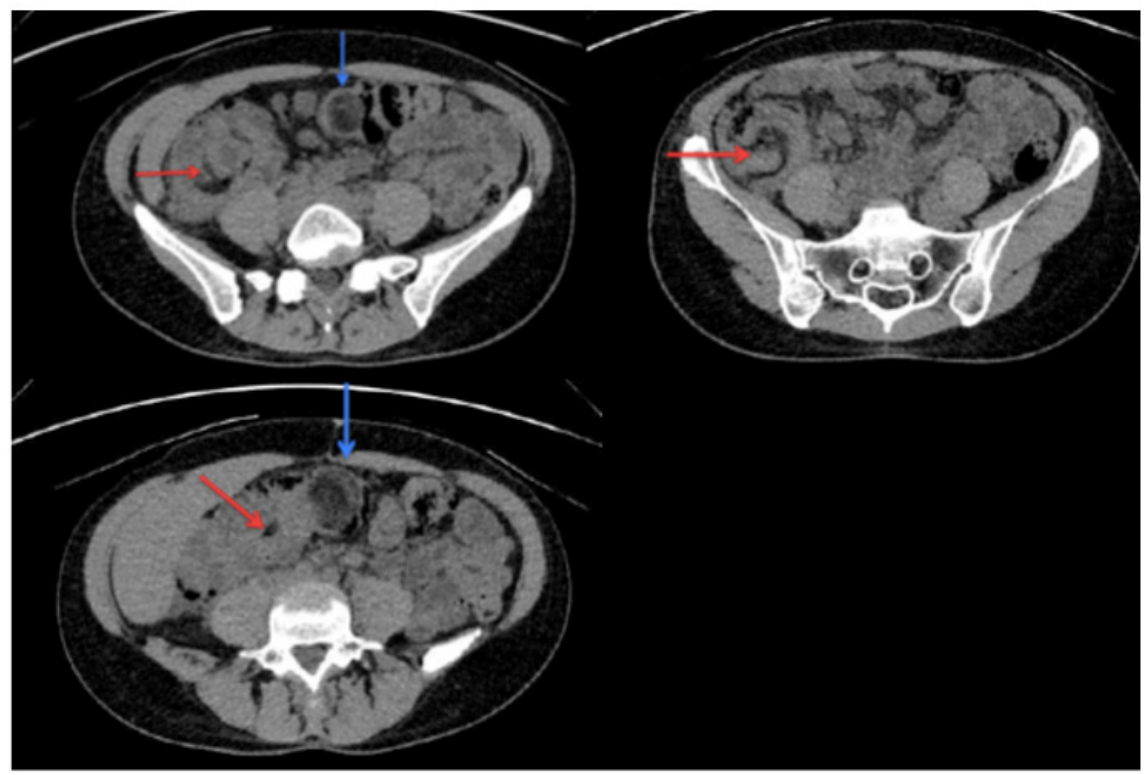

Figura 1. TAC simple de abdomen. La flecha roja señala el lipoma que actúa como punta de lanza a la cabeza de la intususcepción. La flecha azul evidencia la lesión en diana característica, la cual avanza hasta el colon transverso. 


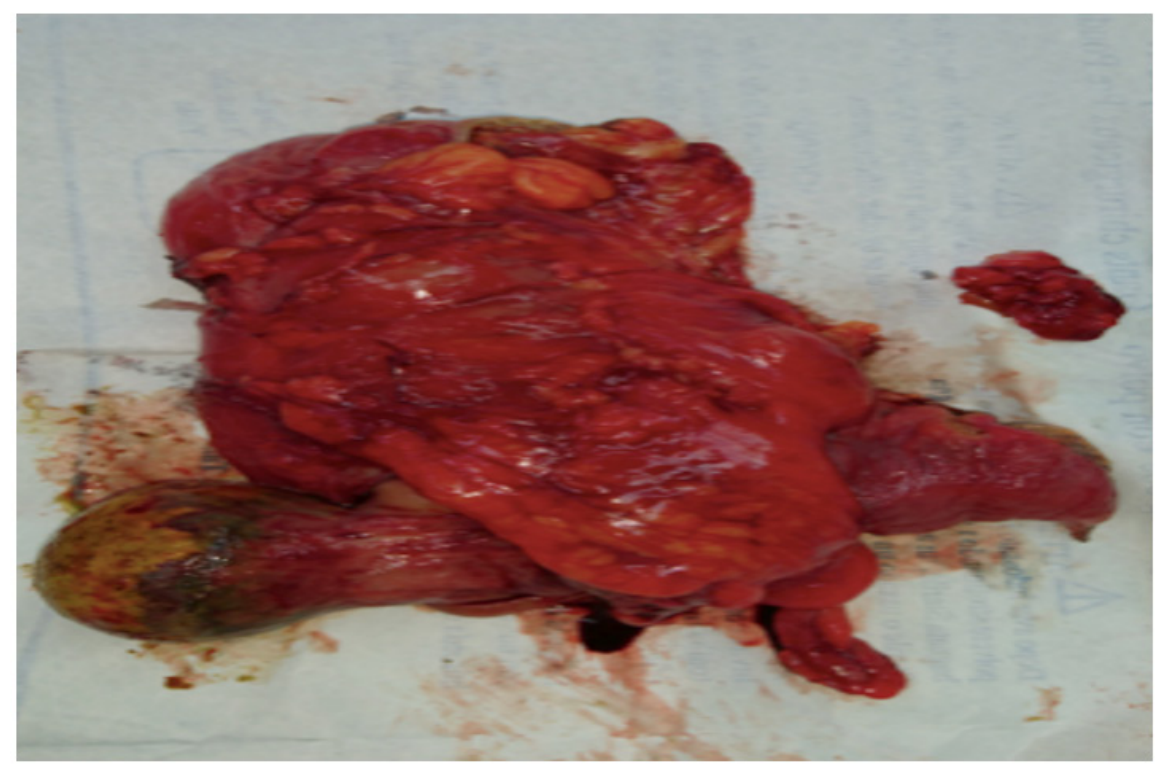

Figura 2. Espécimen resecado. La flecha señala el lipoma que actuó como punta de lanza para producir la intususcepción

Los síntomas y signos más comunes son dolor, náuseas, vómito, diarrea y sangre en las heces (en jalea de "grosella"), y menos común constipación y masa abdominal.

\section{Discusión}

La intususcepción se clasifica según su origen: en el intestino delgado (enteroentérica, ileocólica e ileocecal) o en el colon (colocolónica), y según su etiología o su presentación. Son de especial cuidado las ileocecales, debido al efecto obstructivo que produce la válvula cecal (므). El tipo de intususcepción más común es la entérica y la menos frecuente la colónica ().

Las intususcepciones colónicas relacionadas con tumores se asocian a malignidad en un 65 a $70 \%$, mientras que las de intestino delgado solo se asocian a malignidad en un 30 a 35 \% (4). Las estructuras superiores, específicamente esófago, estómago y duodeno, raramente se ven involucradas debido a su falta de movilidad, redundancia y fijación anatómica. Es usual que la intususcepción ocurra entre un segmento móvil del intestino que ingresa en un segmento relativamente fijo al retroperitoneo o por adherencias ().

La presentación clínica puede ser aguda y en la población adulta con frecuencia los síntomas son subagudos -como en el caso de descrito-, crónicos o intermitentes con recurrencia, en concordancia con la obstrucción parcial e intermitente que producen $(\underline{6}, \underline{9})$.

Los síntomas y signos más comunes son dolor, náuseas, vómito, diarrea y sangre en las heces (en jalea de "grosella"), y menos común constipación y masa abdominal $(\underline{6}, \underline{10})$. Al examen físico se puede evidenciar el signo de Dance (fosa iliaca derecha que se palpa vacía) con frecuencia asociado a la palpación de masa en flanco e hipocondrio derecho, hallazgo no descrito en el examen físico de la paciente $(\underline{4}, \underline{9})$.

Los análisis de laboratorio no son específicos, pudiéndose evidenciar leve leucocitosis con desviación hacia la izquierda y elevación inespecífica de la proteína C reactiva (11). La paciente presentaba leucocitosis leve sin presentar desviación de la curva hacia la izquierda pues no presentaba neutrofilia. 
El estudio de imagen más sensible para el diagnóstico es la tomografía contrastada, con precisión entre 58 y $100 \%$, y es el estándar de oro. Con esta puede evidenciarse la lesión en diana, un área central densa con un halo de menor atenuación que corresponden, respectivamente, al intususceptum y al intususcepiens edematizado.
El estudio de imagen más sensible para el diagnóstico es la tomografía contrastada que tiene una precisión entre 58 y $100 \%$, por lo que es el estándar de oro. Con esta se puede evidenciar la lesión en diana, que consiste en un área central densa con un halo de menor atenuación que corresponden, respectivamente, al intususceptum y al intususcepiens edematizado $(\underline{4}, \underline{10}, \underline{12})$ y en el plano sagital se puede evidenciar el signo de la "salchicha" o pseudoriñon. Estas lesiones, aunque altamente sugestivas, no son patognomónicas. La tomografía puede dar información sobre la localización, nivel y causa de la intususcepción, además sobre posibles complicaciones como signos de obstrucción intestinal, isquemia intestinal, liquido libre, adenopatías y posibles metástasis $(\underline{8}, \underline{13}, 14)$. Con el aumento en la disponibilidad de la tomografía se ha evidenciado un aumento en la prevalencia de las intususcepciones transitorias asintomáticas que suelen resolver espontáneamente $(\underline{8}, \underline{15})$.

La ultrasonografía se ha descrito como una opción diagnóstica en la población pediátrica, ademas ofrece información sobre la circulación enteral. Sin embargo, presenta serias limitaciones en pacientes obesos o con aumento de gas intestinal; además es operador dependiente. Lo anterior, más el advenimiento y mayor disponibilidad de técnicas de imagen con mejor rendimiento, hacen que la ultrasonografía y la radiografía abdominal hayan quedado relegadas (4, 7).

El diagnóstico de la paciente del caso se realizó con una tomografía simple de abdomen con protocolo decúbito prono (uroTAC), pues la sospecha diagnóstica distaba de la causa real de los síntomas. Sin embargo, mediante esta técnica se pudo realizar un diagnóstico certero de intususcepción y su causa subyacente. Debe quedar claro que, ante la sospecha de intususcepción, siempre se debe realizar una tomografía de abdomen contrastada como se mencionó previamente. Esto ilustra por qué, a menudo, el diagnóstico se retrasa y es frecuente que se realice un diagnóstico preoperatorio de obstrucción intestinal y la intususcepción sea encontrada en el acto quirúrgico.

En los adultos, el manejo quirúrgico sigue siendo la elección en más del $90 \%$ de los casos. La cirugía puede modificarse y afectarse por factores tales como localización, obstrucción intestinal aguda subyacente, lesión intestinal isquémica, tamaño de la resección, probabilidad de malignidad de la lesión, entre otras. Anteriormente, la laparotomía con resección en bloque era el tratamiento más empleado; sin embargo, las técnicas actuales están más a favor de realizar una reducción previa de la intususcepción y realizar posteriormente la resección de la lesión y la anastomosis. Este manejo cobra mayor importancia cuando la resección en bloque implica resecar una gran porción de intestino o se sospecha una lesión benigna $(\underline{8}, \underline{13}, 15)$.

Quienes se encuentran a favor de la resección en bloque argumentan que la manipulación del segmento invaginado y de su recipiente puede causar embolización de células cancerígenas a través del sistema venoso en caso de lesiones malignas; además, la manipulación de los segmentos intestinales edematizados y friables, podría aumentar la probabilidad de perforación intestinal produciendo la liberación de material intestinal y células cancerígenas al peritoneo, lo que podría llevar a mayor diseminación. Sin embargo, en la literatura no hay evidencia sólida que respalde estas teorías, solo se cuenta con reportes anecdóticos. Se propone un abordaje selectivo de reducción y resección, tal como se desarrolló en el caso clínico presentado, aunque en nuestro caso fue necesaria la conversión a cirugía abierta para la adecuada realización de la colectomía. El manejo laparoscópico total es factible con las técnicas y los recursos adecuados, logrando tasas de éxito similares a la cirugía convencional $(\underline{8}, \underline{11}, \underline{13-15})$. 
Las técnicas actuales están más a favor de realizar una reducción previa de la intususcepción y realizar posteriormente la resección de la lesión y la anastomosis.

\section{Conclusiones}

La intususcepción en adultos es una entidad relativamente rara y en la cual la causa idiopática es la excepción y las lesiones que actúan como punta de lanza son la regla. Su presentación clínica es inespecífica lo que dificulta la realización de un diagnóstico oportuno, requiriendo una alta sospecha para solicitar las ayudas diagnósticas que permitan identificarla y proceder a su manejo. El tratamiento es principalmente quirúrgico.

\section{Responsabilidades éticas}

Protección de personas y animales

Los autores declaran que para esta investigación no se han realizado experimentos en seres humanos ni en animales.

Confidencialidad de los datos

Los autores declaran que han seguido los protocolos de su centro de trabajo sobre la publicación de datos de pacientes.

Derecho a la privacidad y consentimiento informado

Los autores declaran que en este artículo no aparecen datos de pacientes.

\section{Financiamiento}

No se recibió patrocinio de ningún tipo para llevar a cabo este artículo.

\section{Conflicto de intereses}

Los autores declaran no tener ningún conflicto de intereses.

\section{Bibliografía}

1. Loo GH, Mohamad Abu Zeid WM, Lim SL, Ismail AM. Rare presentation of idiopathic duodenoduodenal intussusception. Ann R Coll Surg Engl. 2017;99 (6):e188-e188-90.

2. Tate JE, Mwenda JM, Armah G, Jani B, Omore R, Ademe A, et al. Evaluation of intussusception after monovalent rotavirus vaccination in Africa. N Engl J Med. 2018;378 (16):1521-8.

3. Amr MA, Polites SF, Alzghari M, Onkendi EO, Grotz TE, Zielinski MD. Intussusception in adults and the role of evolving computed tomography technology. Am J Surg. 2015;209 (3):580-580-3.

4. Al-Radaideh AM, Omari HZ, Bani-Hani KE. Adult intussusception: A 14-year retrospective study of clinical assessment and computed tomography diagnosis. Acta Gastroenterol Belg. 2018;81 (3):367-367-72.

5. Mocanu SN, Botey Fernández M, Simó Alari FB, García San Pedro Á. Acute anterograde intussusception as a late complication of distal gastric bypass. J Med Case Rep. 2018;(9):rjy248-rjy248.

6. Knight AM, Haurani C, Hollenbeck A, Greene F, Mostafa G. Intussusception in the adult population: a challenge to surgeons. Am Surg. 2017;83 (4):e131-e131-3. 
7. de Clerck F, Vanderstraeten E, De Vos M, Van Steenkiste C. Adult intussusception: 10-year experience in two Belgian centres. Acta Gastroenterol Belg. 2016;79 (3):301-301-8.

8. Marsicovetere P, Ivatury SJ, White B, Holubar SD. Intestinal intussusception: etiology, diagnosis, and treatment. Clin Colon Rectal Surg. 2017;30 (1):30-30-9.

9. Martín-Lorenzo JG, Torralba-Martinez A, Lirón-Ruiz R, Flores-Pastor B, Miguel-Perelló J, Aguilar-Jimenez J, et al. Intestinal invagination in adults: preoperative diagnosis and management. Int J Colorectal Dis. 2004;19 (1):68-68-72.

10. Barbuto AF, Masullo LN. Intussusception mimicking acute appendicitis in an adult. J Emerg Med. 2018;55 (5):714-714-5.

11. McKay R. Ileocecal intussusception in an adult: the laparoscopic approach. JSLS. 2006;10 (2):250-250-3.

12. Gueye ML, Sarr ISS, Gueye MN, Thiam O, Seck M, Toure AO, et al. Adult ileocecal intussusception induced by adenomatous ileal polyp: case report and literature review. Int J Surg Case Rep. 2018;2018 (9):rjy256-rjy256.

13. Wang N, Cui XY, Liu Y, Long J, Xu YH, Guo RX, et al. Adult intussusception: a retrospective review of 41 cases. World J. Gastroenterol. 2009;15 (26):33033303-8.

14. Ciftci F. Diagnosis and treatment of intestinal intussusception in adults: a rare experience for surgeons. Int J Clin Exp Med. 2015;8 (6):10001-10001-5.

15. Shenoy S. Adult intussusception: A case series and review. World J Gastrointest Endosc. 2017;9 (5):220-220-7. 